\title{
Eficácia dos testes térmico e elétrico no registro do status pulpar: um estudo in vivo
}

\author{
Effectiveness of thermal and electric tests to register pulp status: in vivo study
}

\section{Thalita Silva}

Mestre em Endodontia, Universidade Estácio de Sá (Unesa), Rio de Janeiro, RJ, Brasil

\section{Sabrina de Castro Brasil}

Doutoranda em Endodontia, Universidade Estácio de Sá (Unesa), Rio de Janeiro, RJ, Brasil

Professora de Endodontia do Centro Universitário Serra dos Órgãos (FESO), Teresópolis, RJ, Brasil

Luci Armada-Dias

Doutora em Biologia, Ueri, Rio de Janeiro, RJ, Brasil

Professora de Fisiologia, Universidade Estácio de Sá (Unesa), Rio de Janeiro, RJ, Brasil

Luciana Armada

Doutora em Fisiopatologia, Uerj, Rio de Janeiro, RJ, Brasil

Professora do Programa de Pós-graduação em Odontologia, Universidade Estácio de Sá (Unesa), Rio de Janeiro, RJ, Brasil

- Os autores declaram que não há conflito de interesse.

\section{Resumo}

Objetivo: Verificar a eficácia dos testes pulpares térmico e elétrico em registrar o status pulpar. Material e Métodos: Foram testados 30 dentes indicados para tratamento endodôntico e com status pulpar desconhecido. Os resultados obtidos foram comparados com a inspeção pulpar direta para a determinação dos números de verdadeiro positivo, falso positivo, verdadeiro negativo e falso negativo. Resultados: A partir dos valores encontrados foram estabelecidos a sensibilidade $(0,94)$, especificidade (elétrico e calor $=0,58$ e frio $=0,75$ ), valor preditivo negativo (elétrico e calor $=0,77$, frio $=0,85$ ), valor preditivo positivo (elétrico e calor $=0,87$, frio $=0,9$ ) e acurácia ( $80 \%$ elétrico e calor e $87 \%$ frio) de cada teste. Conclusão: 0 teste frio apresentou superioridade aos demais testes na maioria dos parâmetros avaliados, sendo considerado o mais eficaz ao avaliar o status pulpar.

Palavras-chave: testes pulpares; diagnóstico; doenças pulpares.

\section{Abstract}

Objective: To verify the effectiveness of thermal and electric pulp test to register the pulp status. Material and Methods: We tested 30 teeth indicated for endodontic treatment and unknown pulp status. The results were compared with the direct pulp inspection to determine the positive real numbers, false positive, true negative and false negative. Results: From the found values were established sensitivity $(0.94)$, specificity (electric, heat and cold $=0.58=0.75$ ), negative predictive value (electric and heat $=0.77$, cold $=0,85$ ), positive predictive value (electric and heat $=0.87$, cold $=0.9$ ) and accuracy ( $80 \%$ electric and heat, and cold $87 \%$ ) of each test. Conclusion: The cold test showed superiority to other tests in most parameters assessed, and is considered the most effective in assessing the pulp status.

Keywords: pulp testing; diagnosis; pulpal diseases.

\section{Introdução}

O s testes pulpares (testes de vitalidade e de sensibilidade) são utilizados como recursos suplementares do exame clínico, para auxiliar no diagnóstico das alterações pulpares. ${ }^{1-4}$ Os testes de vitalidade pulpar (fluxometria laser doppler e oximetria de pulso) indicam a existência de uma circulação vascular adequada, podendo ser usados em conjunto com a sondagem periodontal, percussão, palpação, mobilidade, transiluminação e testes anestésicos, no processo de diagnóstico., ${ }^{4,5}$

Os testes de sensibilidade pulpar são fundamentados na função da inervação, ou seja, sinalização de dano presente ou eminente e na resposta exagerada para indicar baixo limiar de excitabilidade das fibras nervosas. ${ }^{4,6}$ Os mais comumente usados são aqueles que estimulam a polpa através do movimento de fluídos nos túbulos dentinários por mudanças na temperatura ou por corrente elétrica. ${ }^{1}$

Os testes de sensibilidade pulpar não são capazes de verificar a existência de circulação vascular. Porém, a literatura aponta que clinicamente estes são os testes mais utilizados para verificar o status pulpar devido à simplicidade e ao baixo custo da técnica. ${ }^{4}$ Diversos estudos ${ }^{2,7-9}$ avaliaram a habilidade dos testes de sensibilidade em registrar o status pulpar, mas ainda existe uma necessidade de obter informações mais específicas (sensibilidade, especificidade, valores preditivos negativo e positivo) sobre estes. Portanto, o objetivo deste estudo foi verificar a eficácia dos testes pulpares térmicos e elétrico em registrar o status pulpar.

\section{Material e Métodos}

Participaram do estudo 30 pacientes de ambos os sexos, encaminhados para tratamento nas clínicas de graduação e especialização em Endodontia da Universidade Estácio de Sá. Os participantes foram informados sobre o conteúdo da pesquisa e assinaram o termo de consentimento livre e esclarecido. Os procedimentos de anamnese, exame clínico e exame radiográfico foram realizados em seguida. O projeto de pesquisa foi aprovado pelo Comitê de Ética em Pesquisa da Universidade Estácio de Sá (CAAE-0129.0.308.000-11).

Foram excluídos do estudo os pacientes portadores de doença periodontal, usuários de marcapasso, com histórico de tratamento ortodôntico ou traumatismo dentário recente, usuários de analgésicos, tranquilizantes ou alcoolizados. Em relação aos dentes, foram excluídos aqueles que apresentavam: coroa total metálica, cavidade pulpar atrésica, pulpotomia, 
maior espessura de esmalte e dentina, alterações degenerativas, fenômenos reacionais da polpa e rizogênese incompleta.

Após esta etapa, foram realizados por um único endodontista os testes de sensibilidade pulpar (elétrico e térmico). Em cada paciente foi avaliado um dente com indicação prévia de tratamento endodôntico (grupo de estudo) e com status pulpar desconhecido pelo examinador.

Para avaliar a qualidade de execução da técnica e determinar a reação de cada indivíduo aos estímulos, também foram testados dentes unirradiculares do quadrante adjacente com status de normalidade comprovado por exames clínico (ausência de lesão cariosa, doença periodontal e restauração extensa) e radiológico (ausência de espessamento do espaço do ligamento periodontal).

Antes de cada procedimento, os elementos dentários foram secos com jato de ar e submetidos ao isolamento relativo com auxílio do sugador, gaze seca e algodão. Os testes foram executados nas faces: cervical de incisivos, cervical e oclusal de pré-molares, cervical e oclusal (cúspide mésio-vestibular) de molares. Todos os testes foram realizados três vezes em cada elemento dentário com intervalo de 2 minutos.

No teste frio foi utilizado o gás refrigerante (Tetrafluoretano) Endo-Ice (Maquira Dental, Maringá, Paraná, Brasil), aplicado com auxílio de um cotonete na superfície dentária. Para realizar o teste pelo calor, o bastão de guta-percha (Dentsply Maillefer, Petropólis, Rio de Janeiro, Brasil) foi aquecido em lamparina (Jon, Vila Esperança, São Paulo, Brasil) até adquirir um aspecto brilhante. Antes da aplicação a superfície dentária foi lubrificada com vaselina (Fábrica Ipiranga, Vila Prudente, São Paulo, Brasil).

O Aparelho Pulp-Tester (ODOUS de Deus, Belo Horizonte, Minas Gerais, Brasil) foi utilizado no teste elétrico. A ponta do aplicador foi revestida com pasta profilática Herjos (Vigodent, Rio de Janeiro, RJ, Brasil) e posicionada na superfície dentária, que também recebeu a mesma pasta. Os pacientes foram orientados a utilizar o polegar e o indicador sobre o condutor elétrico para completar o circuito e a liberar o clipe quando detectado calor, dormência ou dor. A taxa de condução elétrica foi aumentada lentamente, sendo padronizada uma leitura numérica que foi utilizada como valor controle no paciente. Foi considerada a escala de reações de acordo com as orientações do fabricante.

Após os testes de sensibilidade pulpar, os dentes do grupo de estudo foram submetidos ao isolamento absoluto para a realização do acesso coronário. A abertura da câmara pulpar permitiu a inspeção visual direta para a determinação do status da polpa dentária (vitalidade ou necrose). Foram classificados como vitais os casos de sangramento do tecido pulpar e como necroses aqueles que não apresentaram sangramento e/ou o tecido pulpar encontrava-se destruído.

Os resultados obtidos através dos testes de sensibilidade foram comparados com a inspeção pulpar direta. Foram considerados positivos para a doença os dentes com necrose pulpar e negativos para a doença aqueles que apresentavam a polpa vital. Os números de verdadeiro positivo (VP), falso positivo (FP), verdadeiro negativo (VN) e falso negativo (FN) foram calculados para cada método.

Com base nestes resultados a sensibilidade (capacidade de um teste para identificar os dentes que realmente estão doentes), especificidade (capacidade de um teste para identificar os dentes sem doença), valor preditivo positivo (probabilidade de um teste com resultado positivo realmente representar um dente doente), valor preditivo negativo (probabilidade de um teste com resultado negativo realmente representar um dente livre da doença) e acurácia (verifica a taxa de concordância entre o teste de sensibilidade e a inspeção pulpar direta) foram calculados através das fórmulas observadas no quadro 1.

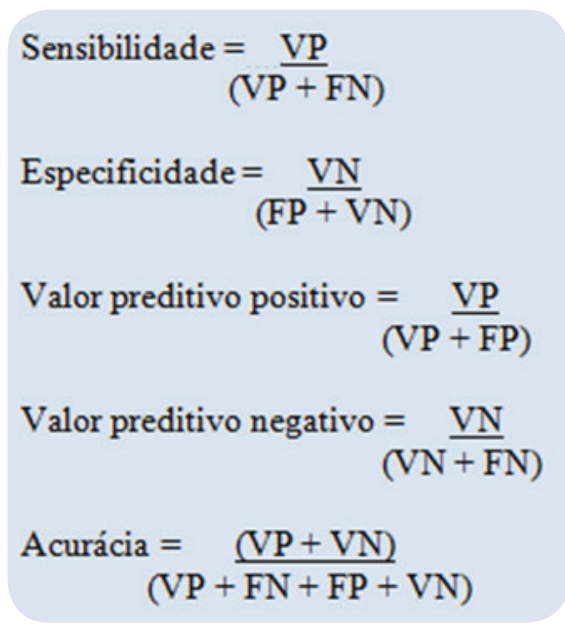

Quadro 1. Fórmulas para o cálculo da sensibilidade, especificidade, valor preditivo positivo, valor preditivo negativo e acurácia 


\section{Resultados}

O estudo foi constituído por 30 pacientes, sendo 15 do gênero masculino e 15 do feminino. A idade média dos indivíduos foi $36,7 \pm 10,6$ (média \pm desvio padrão), sendo a idade mínima de 21 anos e a máxima de 60 anos.

Cada paciente teve dois dentes submetidos aos testes elétrico e térmico resultando em um total de 60 dentes avaliados (30 dentes do grupo de estudo e 30 dentes com status de normalidade, testados apenas para determinar a reação de cada indivíduo). A tabela 1 apresenta a distribuição dos elementos dentários do grupo de estudo.

Após os testes de sensibilidade pulpar, os dentes do grupo de estudo foram submetidos à abertura da câmara pulpar que permitiu o estabelecimento do status da polpa dentária. A prevalência de doença neste grupo foi de $60 \%$ (18 dentes com necrose e 12 com vitalidade).

Os números de casos VP, FP, VN e FN foram determinados para cada teste de sensibilidade através da comparação dos resultados com a inspeção direta da polpa.

O teste elétrico identificou 17 dentes com necrose (um dente necrosado apresentou reação de sensibilidade) e sete com vitalidade (cinco dentes vitais não apresentaram reação de sensibilidade). O teste frio identificou 17 dentes com necrose (um dente necrosado apresentou reação de sensibilidade) e nove com vitalidade (três dentes vitais não apresentaram reação de sensibilidade). $O$ teste pelo calor identificou 17 dentes com necrose (um dente necrosado apresentou reação de sensibilidade) e sete com vitalidade (cinco dentes vitais não apresentaram reação de sensibilidade).

A partir destes valores foram determinadas para cada tipo de teste: sensibilidade, especificidade, valor preditivo positivo e valor preditivo negativo (Tabela 2 ).

Através dos resultados de sensibilidade e especificidade, tanto no teste pelo calor como no elétrico, foi possível verificar que $94 \%$ dos dentes com necrose e $58 \%$ dos dentes com vitalidade foram identificados corretamente. Já os valores preditivos negativo e positivo determinaram que, com estes testes, existe a probabilidade de $77 \%$ dos resultados não sensíveis representarem necrose pulpar e de $87 \%$ dos resultados sensíveis representarem vitalidade pulpar. A acurácia verificou $80 \%$ de concordância com a inspeção visual direta da polpa.

Os resultados de sensibilidade e especificidade no teste frio determinaram que $94 \%$ dos dentes com necrose e $75 \%$ dos dentes com vitalidade foram identificados corretamente. Já os valores preditivos negativo e positivo verificaram que, com este teste, existe a probabilidade de $85 \%$ dos resultados não sensíveis representarem necrose pulpar e de $90 \%$ dos resultados sensíveis representarem vitalidade pulpar. A acurácia verificou $87 \%$ de concordância com a inspeção visual direta da polpa.

\section{Discussão}

O tecido pulpar não pode ser diretamente inspecionado antes do tratamento endodôntico. Portanto, métodos indiretos devem ser usados para determinar o status pulpar. Os testes mais comumente usados são os testes de sensibilidade térmica e elétrica que estimulam a polpa através do movimento de fluídos nos túbulos dentinários. ${ }^{10}$ Por esta razão, estes testes foram executados neste estudo. Roberts et al. ${ }^{11}$, Elminguid Yu1 ${ }^{2}$, Levin et al..$^{13}$ e Weisleder et al. ${ }^{10}$ observaram que os testes elétricos e térmicos são de grande valia para o correto diagnóstico.

A maioria dos trabalhos ${ }^{3,6,8,14-17}$ de validação dos testes de sensibilidade pulpar compara apenas os testes elétrico e frio não avaliando a eficácia do teste pelo calor. O presente estudo, considerando a importância destes três testes de sensibilidade no diagnóstico, verificou em todos os parâmetros de sensibilidade, especificidade, valor preditivo positivo, valor preditivo negativo e acurácia.

Nesta pesquisa foram estabelecidas idades mínima (21 anos) e máxima (50 anos) para que fossem evitados casos de rizogênese incompleta e de canais calcificados ou atresiados. ${ }^{18,19}$

O presente estudo realizou testes de sensibilidade pulpar em 30 pacientes. A seleção dos indivíduos e dentes foi baseada em critérios de exclusão semelhantes àqueles realizados por Pertesson et al. ${ }^{2}$ e Susarla et al. ${ }^{6}$ que também realizaram testes elétrico e térmicos.

Os testes de sensibilidade foram realizados na cervical dos dentes anteriores, por haver uma menor espessura de esmalte nessas áreas, concordando com a metodologia utilizada por Bender et al. ${ }^{20} \mathrm{e} \mathrm{Jafarzadeh,} \mathrm{Abbot}{ }^{4}$ que analisaram a diferença na posição da ponta do eletrodo nos testes elétricos de dentes anteriores.

No presente estudo, 30 dentes com status de normalidade também foram testados. Estes elementos dentários apresentaram resultados sensíveis aos testes comprovando a utilização adequada da técnica e a capacidade do paciente em identificar os estímulos. Foram selecionados apenas dentes unirradiculares por serem considerados mais confiáveis. Em molares existe a possibilidade de haver necrose pulpar em uma raiz e vitalidade em outra ${ }^{20}$.

A prevalência de doença neste estudo foi de $60 \%$ (18 em 30 dentes), divergindo de Petersson et al. ${ }^{2}$, que apresentou $39 \%$ de prevalência. Este percentual elevado possivelmente deve-se ao fato de todos os dentes do grupo de estudo terem sido encaminhados por apresentarem necessidade de tratamento endodôntico.

A sensibilidade dos testes elétrico, frio e calor $(0,94)$ foi superior a encontrada por Petersson et al. ${ }^{2}$ para os mesmos testes pulpares (elétrico: 0,72, frio: 0,83, calor: 0,86). A especificidade foi o parâmetro que apresentou os valores de todos os testes inferiores em relação à literatura. ${ }^{2,16}$ Os valores preditivos positivo e negativo foram compatíveis com os estudos que utilizaram metodologia semelhante., ${ }^{2,18} \mathrm{~A}$ acurácia foi de $80 \%$ para os testes elétrico e pelo calor e $87 \%$ para o teste frio. Os percentuais dos testes elétrico e frio estão de acordo com a literatura. ${ }^{2,16,18} \mathrm{O}$ teste pelo calor apresentou valor superior ao encontrado por Petersson et al. ${ }^{2}$

Acredita-se que esta elevada prevalência de doença no grupo de estudo tenha refletido em diferenças entre os nossos resultados e aqueles encontrados na literatura. 
Tabela 1. Distribuição dos dentes do grupo de estudo

\begin{tabular}{|c|c|c|c|c|}
\hline Arcada & Molares & Pré-molares & Incisivos e caninos & Total \\
\hline Maxilar & 05 & 07 & 04 & 16 \\
\hline Mandibular & 09 & 03 & 02 & 14 \\
\hline Total & 14 & 10 & 06 & 30 \\
\hline
\end{tabular}

Tabela 2. Sensibilidade, Especificidade, Valor Preditivo Negativo (VPN) e Valor Preditivo Positivo (VPP) dos Testes Elétrico, Frio e Calor

\begin{tabular}{|c|c|c|c|c|} 
Teste & Sensibilidade & Especificidade & VPN & VPP \\
\hline Elétrico & 0,94 & 0,58 & 0,77 & 0,87 \\
\hline Frio & 0,94 & 0,75 & 0,85 & 0,9 \\
\hline Calor & 0,94 & 0,58 & 0,77 & 0,87 \\
\hline
\end{tabular}

\section{Conclusão}

A partir deste estudo foi possível concluir que o teste frio apresentou superioridade aos demais testes na maioria dos parâmetros avaliados, sendo considerado o mais eficaz ao avaliar o status pulpar em dentes vitais e necrosados.

\section{Referências ::}

1. Lopes HP, Siqueira JR JF. Endodontia Biologia e Técnica. Rio de Janeiro: Guanabara Koogan, 2015.

2. Petersson K, Soderstrom C, Kiani-Anaraki M, Levy G. Evaluation of the ability of thermal and electrical tests to register pulp vitality. J Endod Dent Traumatol. 1999;15:127-31.

3. Gopikrishna V, Tinagupta K, Kandasway D. Comparison of electrical, thermal, and pulse oximetry. Methods for assessing pulp vitality in recently traumatized teeth. Clinical Research. 2007;33:47-9.

4. Jafarzadeh H, Abbot PV. Review of the pulp sensibility tests. Part I: General information and thermal tests. J Int. Endodontics. 2010;43:738-62.

5. Bastos JV, Goulard EM, de Souza Côrtes MI. Pulpal response to sensibility tests after traumatic dental injuries in permanent teeth. Dental Traumatol. 2014;30(3):188-92.

6. Susarla MS, Hsiao-HU, White R. Use of cold test as a measure of pulpal anesthesia during endodontic therapy: A randomized, blinded, placebo-controlled. Rev Clinical Trial. 2007;3:4-7.

7. Pantera EA, Anderson RW, Pantera CT. Reliability of electrical pulp testing after pulpal testing with dichlorodifluoromethane. J Endodondontics. 1993;19:12-4.

8. Farid H, Khan FR, Pasha L, Shinwari MS. Are pulp sensibility tests still sensible? J Ayub Med Coll Abbotabad. 2015;27(4):874-7.

9. Trope \& Debelian Dental Pulp Testing. J Internacional Dentistry. 2005;52:1-12.

10. Weisleder R, Yamauchi S, Caplan JD, Trope M, Teixeira BF. The validity of pulp testing: a clinical study. J Dent Assoc. 2009;140:1013-7.
11. Roberts C, Torgerson DJ, Walter P. Pulp sensibity tests. J Int. Endodontics. 2002;16:28-31.

12. Elminguid AA \& YU D. Dental pulp neurophysiology: party 1.Clinical and Diagnostic Implications. JCAD. 2009;75:55-8.

13. Levin LG, Law AS, Holland GR, Abbott PV. Identify and define all diagnostic terms for pulpal health and disease states. J Endodontics. 2009;35(12):1645-57.

14. Jespersen JJ, Hellsein J, Williamson A, Johnson WT, Qian F. Evaluation of dental pulp sensibility tests in a clinical setting. J Endod. 2014;40(3):351-14.

15. Jafarzadeh H, Rosenberg PA. Pulse oximetry review of potential aid in endodontic diagnosis. J Int. Endodontics. 2009;35:329-33.

16. Chen E, Abbot PV. Evalution of accuracy, reability, and repeatability of five dental pulp testes. J Int. Endodontics. 2009;37:1619-23.

17. Lin J, Chandler N, Purton D, Monteith B. Appropriate electrode placement site for electric pulp testing first molar teeth. J Endod. 2007;33(11):1296-8.

18. Cohen ME, Hyman J. The predictive value of endodontic diagnostic tests. Oral Surg Oral Med Oral Pathol. 1984;58:343-9.

19. Fuss Z, Trowbridge H, Bender IB, Rickoff B, Solomon S. Assessment of reliability of electrical and thermal pulp testing agents. J Int. Endodontics. 1986;12:301-5.

20. Bender IB. Pulpal pain diagnosis- a review. J Endodontics. 1989;26(3):175-179.

Recebido em: 28/03/2016 / Aprovado em: 27/04/2016

Sabrina de Castro Brasil

Rua Presidente Pedreira, 153/602- Ingá

Niterói/RJ, Brasil - CEP: 24210-470

E-mail: sabrinacbrasil@hotmail.com

Agradecimentos

À Universidade Estácio de Sá. 